PROCEEDINGS OF THE

AMERICAN MATHEMATICAL SOCIETY

Volume 140, Number 8, August 2012, Pages 2893-2902

S 0002-9939(2011)11103-1

Article electronically published on June 2, 2011

\title{
THE DIAGONAL REFLECTION PRINCIPLE
}

\author{
SEAN COX
}

(Communicated by Julia Knight)

\begin{abstract}
We introduce a highly simultaneous version of stationary set reflection, called the Diagonal Reflection Principle (DRP). We prove that $P F A^{+\omega_{1}}$ implies DRP, and DRP in turn implies that the nonstationary ideal on $[\theta]^{\omega}$ condenses correctly for many structures. We also prove that MM implies a weaker version of DRP, which in turn implies that the nonstationary ideal on $\theta \cap \operatorname{cof}(\omega)$ condenses correctly for many structures.
\end{abstract}

\section{INTRODUCTION}

Stationary set reflection is a compactness property that many large cardinals possess. For a fixed cardinal $\kappa$ and $S \subset \kappa$, we say that $S$ reflects at $\gamma$ if and only if $S \cap \gamma$ is stationary in $\gamma$, and that $S$ reflects if and only if there is a $\gamma<\kappa$ at which $S$ reflects. For example, if $\kappa$ is a measurable cardinal, then every stationary subset of $\kappa$ reflects. Stationary reflection may also occur at "small" cardinals such as $\omega_{2}$ : Harrington and Shelah [8] proved that "every stationary subset of $\omega_{2} \cap \operatorname{cof}(\omega)$ reflects" is equiconsistent with a Mahlo cardinal.

The requirement that every stationary set reflects can be strengthened by requiring that many stationary sets reflect at the same point; such principles go under the general heading of simultaneous stationary reflection. For example, "every pair of stationary subsets of $\omega_{2} \cap \operatorname{cof}(\omega)$ have a common reflection point" is equiconsistent with a weakly compact cardinal (Magidor [11, Baumgartner [1]).

Strong forcing axioms imply simultaneous stationary reflection. Baumgartner [2] showed that $P F A^{+\omega_{1}}$ implies that for all regular $\theta \geq \omega_{2}$, any $\omega_{1}$-sized collection of stationary subsets of $\theta \cap \operatorname{cof}(\omega)$ has a common reflection point of the smallest possible cofinality (namely, $\omega_{1}$ ) 11 the consistency strength of the latter is not known for $\theta=\omega_{3}$, but it requires at least measurable cardinals of high Mitchell order (see [3]).

Stationary set reflection for the generalized notion of stationarity on subsets of $[\theta]^{\omega}$ gained prominence in [7. In that paper, it was shown that Martin's Maximum implies that every stationary subset of $[\theta]^{\omega}$ reflects to a set of size $\omega_{1}$ and that such stationary reflection implies that $N S_{\omega_{1}}$ is presaturated.

Received by the editors November 11, 2010 and, in revised form, March 2, 2011.

2010 Mathematics Subject Classification. Primary 03E05, 03E50, 03E57.

I thank Matt Foreman, Ralf Schindler, and Martin Zeman for helpful conversations on related topics.

${ }^{1}$ What Baumgartner called $P F A^{+}$in that paper we will call $P F A^{+\omega_{1}}$. 
Foreman [6] showed that Martin's Maximum implies a simultaneous form of stationary reflection for subsets of $\theta \cap \operatorname{cof}(\omega)$. Motivated by this result and his use of condensation-like properties of the nonstationary ideal from that paper, we introduce a highly simultaneous version of stationary set reflection, which we call the Diagonal Reflection Principle (DRP). We prove that DRP follows from $P F A^{+\omega_{1}}$; in fact, just $M A^{+\omega_{1}}(\sigma$-closed) suffices.

DRP, in turn, implies a kind of condensation principle for the nonstationary ideal. Namely, DRP implies that there are many $M$ of cardinality $\omega_{1}$ such that, if $\sigma_{M}: \bar{M} \rightarrow M$ is the inverse of the Mostowski collapse map, then $\bar{M}$ is correct about the stationarity of subsets of $\sigma_{M}^{-1}\left([X]^{\omega}\right)$ (for large $X \in M$ ); in fact, this is equivalent to DRP. Similar condensation principles for ideals related to Chang's Conjecture appeared in Foreman [6], where such principles were used to help produce models with very large cardinals.

We also introduce a weaker version of DRP, which we denote wDRP, and prove that wDRP follows from Martin's Maximum. This provides a factoring of the MM theorem from Foreman [6]. wDRP also implies a form of condensation for the nonstationary ideal.

The paper is organized as follows: Section 2 reviews some background material. Section 3 defines DRP and wDRP and proves the condensation results; this section also discusses the relation between wDRP and the simultaneous notion of stationary reflection which was shown to follow from Martin's Maximum in 6]. Section 4 proves that DRP follows from $P F A^{+\omega_{1}}$. Section 5 proves that wDRP follows from Martin's Maximum. Section 6 concludes with some questions.

\section{BACKGROUND}

$\wp_{\kappa}\left(H_{\theta}\right)$ denotes the set of $M \prec H_{\theta}$ such that $|M|<\kappa$ and $M \cap \kappa \in \kappa$. We consider various classes of $\omega_{1}$-sized structures (more information and generality can be found in [5]). We say that $M$ has uniform cofinality $\omega_{1}$ iff $\operatorname{cof}(\sup (M \cap \kappa))=\omega_{1}$ for every $\kappa \leq \sup (M \cap O R D)$ of uncountable cofinality. $M$ is called internally club of length $\omega_{1}$ iff there is a $\in$-increasing and $\subseteq$-continuous sequence $\left\langle N_{\beta} \mid \beta<\omega_{1}\right\rangle$ of countable elementary substructures of $(M, \in)$ such that $M=\bigcup_{\beta<\omega_{1}} N_{\beta}$ and $N_{\beta} \in M$ for every $\beta<\omega_{1} . M$ is called internally approachable of length $\omega_{1}$ iff there is such a sequence $\vec{N}$ such that every proper initial segment of $\vec{N}$ is an element of $M$. Unif $f_{\omega_{1}}, \mathrm{IC}_{\omega_{1}}$, and $\mathrm{IA}_{\omega_{1}}$ denote, respectively, the classes of structures of uniform cofinality $\omega_{1}$, internally club structures of length $\omega_{1}$, and internally approachable structures of length $\omega_{1}$. Clearly $\mathrm{IA}_{\omega_{1}} \subseteq \mathrm{IC}_{\omega_{1}} \subseteq \mathrm{Unif}_{\omega_{1}}$. It is routine to check that whenever $\theta \geq \omega_{2}$ is regular, then $\operatorname{IA}_{\omega_{1}} \cap \wp_{\omega_{2}}\left(H_{\theta}\right)$ is stationary.

Note that if $\theta \geq \omega_{2}$ is regular, $M \in \mathrm{Unif}_{\omega_{1}}, M \prec H_{\theta}$, and $\sigma_{M}: \bar{M} \rightarrow M$ is the inverse of the Mostowski collapsing map, then:

$\sigma_{M}$ is continuous at points of countable cofinality and $M \cap$ $\sup (M \cap \theta)$ is an $\omega$-club in the ordinal $\sup (M \cap \theta)$.

If $M \in \mathrm{IC}_{\omega_{1}}$, then the sequence from the definition of $\mathrm{IC}_{\omega_{1}}$ witnesses: $M \cap[M]^{\omega}$ contains a closed unbounded subset of $[M]^{\omega}$.

The following is a standard lemma; see for example Lemma 8 of [7]:

Lemma 2.1. Let $S \subset \omega_{1}$ and $R \subset \theta \cap \operatorname{cof}(\omega)$ be stationary sets, where $\theta \geq \omega_{2}$ is a regular cardinal. Then $R \searrow S:=\left\{N \in\left[H_{\theta}\right]^{\omega} \mid \sup (N \cap \theta) \in R\right.$ and $\left.N \cap \omega_{1} \in S\right\}$ is stationary in $\left[H_{\theta}\right]^{\omega}$. 
The following notion was introduced in 4]:

Definition 2.2. Let $Z$ be an uncountable set. A set $R \subset[Z]^{\omega}$ is called projective stationary if and only if for every stationary $T \subset \omega_{1}$, the set $\left\{N \in R \mid N \cap \omega_{1} \in T\right\}$ is stationary.

If $\Gamma$ is a class of posets, $\mathrm{MA}(\Gamma)$ means that whenever $\mathbb{P} \in \Gamma$ and $\left\langle D_{i} \mid i<\omega_{1}\right\rangle$ is a sequence of dense subsets of $\mathbb{P}$, then there is a filter on $\mathbb{P}$ which meets each $D_{i}$. $\mathrm{MA}^{+\alpha}(\Gamma)$ means that whenever $\mathbb{P} \in \Gamma,\left\langle D_{i} \mid i<\omega_{1}\right\rangle$ is a sequence of dense subsets of $\mathbb{P}$, and $\left\langle\dot{S}_{\xi}\right| \xi\langle\alpha\rangle$ is a sequence such that $1_{\mathbb{P}} \Vdash$ " $\dot{S}_{\xi}$ is a stationary subset of $\omega_{1}$ " for all $\xi<\alpha$, then there is a filter $F \subset \mathbb{P}$ which meets every $D_{i}$ and for every $\xi<\alpha:\left(\dot{S}_{\xi}\right)_{F}:=\left\{\beta \mid \exists q \in F q \Vdash \check{\beta} \in \dot{S}_{\xi}\right\}$ is a stationary subset of $\omega_{1}$.

The proof of the next theorem is an easy variation of the proof on page 38 of Woodin 12. For a possibly nontransitive $M \prec\left(H_{\theta}, \in,\{\mathbb{P}\}\right)$, we say that $F$ is an $(M, \mathbb{P})$-generic object if $F$ is a filter on $M \cap \mathbb{P}$ and $D \cap F \neq \emptyset$ for every $D \in M$ which is a dense subset of $\mathbb{P}$. If $F$ is $(M, \mathbb{P})$-generic, then since we require $F \subset M$, we trivially have $D \cap F \cap M \neq \emptyset$ for each dense $D \in M$; sometimes the latter is taken as the definition of " $(M, \mathbb{P})$-generic" if one does not require $F \subset M$.

Theorem 2.3. Assume $M A^{+\alpha}(\{\mathbb{P}\})$, where $\alpha \leq \omega_{1}$ and $\mathbb{P}$ is a poset which preserves $\omega_{1}$. Let $\theta$ be a regular cardinal such that $\mathbb{P} \in H_{\theta}$. Let $\left\langle\dot{S}_{i} \mid i<\alpha\right\rangle$ be a sequence of $\mathbb{P}$ names for stationary subsets of $\omega_{1}$. Then there are stationarily many $M \in P_{\omega_{2}}\left(H_{\theta}\right)$ such that $\omega_{1} \subset M$ and there exists an $(M, \mathbb{P})$-generic object $F$ such that for each $i<\alpha$, the evaluation of $\dot{S}_{i}$ by $F$ is stationary in $V$.

The following definition and lemmas about generic evaluations over nontransitive models are standard, but we include proofs for completeness.

Definition 2.4. Suppose $\mathbb{P}$ is a poset, $2^{2^{|\mathbb{P}|}} \in H_{\Omega}$ (where $\Omega$ is regular), $M \prec\left(H_{\Omega}\right.$, $\in,\{\mathbb{P}\})$, and $F \subset M$ is $(M, \mathbb{P})$-generic. If $\dot{A} \in M$ is a $\mathbb{P}$-name for a subset of the ground model, let $\dot{A}_{F}$ denote the following set:

$$
\{z \mid \exists p \in F p \Vdash \check{z} \in \dot{A}\}
$$

Notice that Definition 2.4 agrees with the notation $\left(\dot{S}_{i}\right)_{F}$ in the definition of $M A^{+\alpha}(\Gamma)$. We briefly point out the relationship between Definition 2.4 and the usual notion of evaluating a name over a transitive model, though we will not need this relationship in this paper. Suppose $M, \dot{A}, \mathbb{P}, \Omega$, and $F$ are as in Definition 2.4 and that additionally $1_{\mathbb{P}} \Vdash \dot{A} \subseteq \check{H}_{\Omega}$. Let $\sigma: \bar{H} \rightarrow H_{\Omega}$ be the inverse of the Mostowski collapse of $M$, and $\bar{F}:=\sigma^{-1} " F$. Then $\bar{F}$ is $\left(\bar{H}, \sigma^{-1}(\mathbb{P})\right)$-generic. Let $\overline{\dot{A}}:=\sigma^{-1}(\dot{A})$. Then $\overline{\dot{A}}_{\bar{F}}$, in the sense of Definition 2.4, is the same as the usual sense of using a generic to evaluate a name over a transitive model. Also, it is easy to check that $\dot{A}_{F} \cap M=\sigma " \overline{\dot{A}}_{\bar{F}}$. If we also assume that $1_{\mathbb{P}} \Vdash \dot{A} \in \check{H}_{\Omega}$, then clearly $\overline{\dot{A}}_{\bar{F}} \in \bar{H}=\operatorname{dom}(\sigma)$ and $\dot{A}_{F}=\sigma\left(\overline{\dot{A}}_{\bar{F}}\right)$.

Lemma 2.5. Let $M, F, \mathbb{P}$, and $\Omega$ be as in Definition 2.4. Suppose $\dot{A} \in M$ is a $\mathbb{P}$-name and $1_{\mathbb{P}}$ forces that $\dot{A} \in \check{H}_{\Omega}$. Then $\dot{A}_{F} \in M$ and is the unique $z \in H_{\Omega}$ such that $(\exists p \in F)(p \Vdash \dot{A}=\check{z})$. Moreover:

(1) if $1_{\mathbb{P}}$ forces $|\dot{A}| \leq|\check{B}|$ for some $B \in M$ such that $B \subseteq M$, then $\dot{A}_{F} \subseteq M$.

(2) If $\phi$ is a $\Sigma_{0}$ formula such that $1_{\mathbb{P}} \Vdash \phi(\dot{A})$, then $\phi\left(\dot{A}_{F}\right)$. 
Proof. Since $1_{\mathbb{P}} \Vdash \dot{A} \in \check{H}_{\Omega}$, then $D:=\left\{p \in \mathbb{P} \mid \exists z \in H_{\Omega} p \Vdash \dot{A}=\check{z}\right\}$ is dense; moreover $D \in M$ so there is a $p \in F \cap D$. Since $p \in M$ and $M \prec\left(H_{\Omega}, \in, \mathbb{P}\right)$, there is a $z \in M$ such that $p \Vdash \dot{A}=\check{z}$. Uniqueness of this $z$ is due to the fact that $F$ is a filter.

If $1_{\mathbb{P}}$ forces $|\dot{A}| \leq|\check{B}|$, where $B \in M$ and $B \subset M$, then there is a name $\dot{h} \in M$ such that $1_{\mathbb{P}} \Vdash \dot{h}: \check{B} \rightarrow_{\text {onto }} \dot{A}$. For each $b \in B$, the set $D_{b}:=\{p \in$ $\mathbb{P} \mid p$ decides $\dot{h}(\breve{b})\}$ is dense and an element of $M$. Since $F$ is an $(M, \mathbb{P})$-generic and $B \subset M$, then $\dot{A}_{F}$ is equal to the collection of $y$ such that for some $p \in F$ and some $b \in B, p \Vdash \dot{h}(\check{b})=\check{y}$. Any such $y$ is definable from $p, b$, and $\dot{h}$ and is thus an element of $M$. So $\dot{A}_{F} \subseteq M$.

Item (2) holds simply because, letting $z:=\dot{A}_{F}$, there is some condition which forces $\phi(\check{z})$; this is a $\Sigma_{0}$ statement in the forcing language, so $\phi(z)$ holds in $V$.

Lemma 2.6. Let $M, F, \mathbb{P}$, and $\Omega$ be as in Definition 2.4. Suppose $\omega_{1} \subset M$, $H \in M$, and $1_{\mathbb{P}}$ forces " $\dot{H} \in I A_{\omega_{1}}$ ". Then $M \cap H \in I A_{\omega_{1}}$. Moreover if $\dot{f} \in M$ is forced to witness that $H \in I A_{\omega_{1}}$, then $\bigcup_{\alpha<\omega_{1}}\left(\dot{f}\lceil\check{\alpha})_{F}\right.$ is a sequence witnessing that $M \cap H \in I A_{\omega_{1}}$.

Proof. Since $H$ is forced to be in $\mathrm{IA}_{\omega_{1}}, M \prec\left(H_{\Omega}, \in,\{\mathbb{P}\}\right)$, and $H \in M$, then there is some name $\dot{f} \in M$ such that $1_{\mathbb{P}} \Vdash$ " $\dot{f}$ witnesses that $\check{H}$ is in $\mathrm{IA}_{\omega_{1}}$ ". Consider any such $\dot{f} \in M$. For each $\alpha<\omega_{1}$, since $\alpha \in M$, then so is the canonical name for $\dot{f} \uparrow \check{\alpha}$. By the definition of internal approachability, $\dot{f} \uparrow \check{\alpha}$ is forced to be an element of $H$; so $\left(\dot{f}\lceil\check{\alpha})_{F} \in M\right.$ by Lemma 2.5. First we check that $\bigcup_{\alpha<\omega_{1}}\left(\dot{f}\lceil\check{\alpha})_{F}\right.$ is a function. Let $\alpha<\alpha^{\prime}<\omega_{1}$, let $h:=\left(\dot{f}\lceil\alpha)_{F}\right.$, and $h^{\prime}:=\left(\dot{f}\left\lceil\alpha^{\prime}\right)_{F}\right.$. There is a condition $p(\in F)$ which forces $\breve{h} \subset \check{h}^{\prime}$; this is a $\Sigma_{0}$ statement (in the forcing language) and so $h \subset h^{\prime}$. Thus $G:=\bigcup_{\alpha<\omega_{1}}(\dot{f} \uparrow \check{\alpha})_{F}$ is a function. To see that $G$ is $\in$-increasing and $\subset$-continuous, it suffices to see that each proper initial segment $\left(\dot{f}\lceil\alpha)_{F}\right.$ has these properties; these are $\Sigma_{0}$ statements about $\left(\dot{f}\lceil\alpha)_{F}\right.$, so the proof is similar to the proof that $G$ is a function. Finally, a density argument shows that $M \cap H$ is a subset of the union of the models enumerated by $\bigcup_{\alpha<\omega_{1}}\left(\dot{f}\lceil\check{\alpha})_{F}\right.$. That this union is contained in $M \cap H$ follows from the fact that each model in the $G$ enumeration is a countable element of $M$ and is thus a subset of $M$.

\section{The Diagonal Reflection Principle (DRP)}

We now define DRP and wDRP.

Definition 3.1. Let $Z$ be a class of $\omega_{1}$-sized sets. The Diagonal Reflection Principle at $\theta$ relative to $Z$, abbreviated $\operatorname{DRP}(\theta, Z)$, is the statement:

There are stationarily many $M \in \wp_{\omega_{2}}\left(H_{\left(\theta^{\omega}\right)^{+}}\right)$such that:

- $M \cap H_{\theta} \in Z$.

- Whenever $R \in M$ is a stationary subset of $[\theta]^{\omega}$, then $R \cap[M \cap \theta]^{\omega}$ is stationary.

Definition 3.2. Let $Z$ be a class of $\omega_{1}$-sized sets. The Weak Diagonal Reflection Principle at $\theta$ relative to $Z$, abbreviated $\operatorname{wDRP}(\theta, Z)$, is the statement:

There are stationarily many $M \in \wp_{\omega_{2}}\left(H_{\left(\theta^{\omega}\right)^{+}}\right)$such that:

- $M \cap H_{\theta} \in Z$.

- Whenever $R \in M$ is a projective stationary subset of $[\theta]^{\omega}$, then $R \cap[M \cap \theta]^{\omega}$ is stationary. 
We thank the anonymous referee for suggesting the present Definition 3.2 , which is simpler than the original.

In this paper, the class $Z$ from Definitions 3.1 and 3.2 will typically be one of Unif $_{\omega_{1}}, \mathrm{IC}_{\omega_{1}}$, or $\mathrm{IA}_{\omega_{1}}$. For suitable $Z, \operatorname{wDRP}(\theta, Z)$ implies reflection for stationary subsets of $\theta \cap \operatorname{cof}(\omega)$ :

Lemma 3.3. Suppose $Z \subset$ Unif $_{\omega_{1}}$. Then $\operatorname{wDRP}(\theta, Z)$ implies there are stationarily many $M \in \wp_{\omega_{2}}\left(H_{\left(\theta^{\omega}\right)+}\right)$ such that $M \cap H_{\theta} \in Z$ and for any $R \in M$ which is a stationary subset of $\theta \cap \operatorname{cof}(\omega)$, then $R$ reflects to $\sup (M \cap \theta)$.

Proof. Let $M$ be any member of the stationary set which witnesses wDRP $(\theta, Z)$. Let $R \in M$ be a stationary subset of $\theta \cap \operatorname{cof}(\omega)$. Let $P_{R}:=\left\{N \in[\theta]^{\omega} \mid \sup (N \cap\right.$ $\theta) \in R$. Then $P_{R} \in M$, and $P_{R}$ is projective stationary by Lemma 2.1. Let $\theta_{M}:=\sup (M \cap \theta)$; note since $Z \subset$ Unif $_{\omega_{1}}$, then $\operatorname{cof}\left(\theta_{M}\right)=\omega_{1}$. Let $c \subset \theta_{M}$ be an arbitrary $\omega$-club; since $Z \subset$ Unif $_{\omega_{1}}$ then $c^{\prime}:=c \cap M$ is an $\omega$-club in $\theta_{M}$. Since $P_{R}$ reflects to $M \cap \theta$, there is an $N \in P_{R}$ such that $N \prec\left(M \cap \theta, \in, c^{\prime}\right)$. Then $\sup (N \cap \theta)$ is an $\omega$-cofinal limit point of $c^{\prime}$, and thus an element of $c^{\prime} \cap R$.

We state two definitions which were used in [6] (though not by name):

Definition 3.4. Let $W$ be a transitive model of $\mathrm{ZFC}^{-}$, and $H \in W$. We say that $\left(N S \uparrow[H]^{\omega}\right)^{W}$ coheres with $N S \uparrow[H]^{\omega}$ if and only if for every $A \in W: W \models$ " $A$ is a stationary subset of $[H]^{\omega}$ " if and only if $A$ is a stationary subset of $[H]^{\omega}$.

Definition 3.5. Let $M \in \wp_{\omega_{2}}\left(H_{\Omega}\right)$, and $H \in M$ be an uncountable set. We say that the nonstationary ideal on $H$ condenses correctly via $M$ if and only if the following holds.

Letting $\sigma_{M}: \bar{M} \rightarrow M$ be the inverse of the Mostowski collapse of $M$ and $\bar{H}:=\sigma_{M}^{-1}(H)$, we have that $\left(N S \uparrow[\bar{H}]^{\omega}\right)^{\bar{M}}$ coheres with $N S \uparrow[\bar{H}]^{\omega}$.

The nonstationary ideal condenses correctly via internally club structures which witness DRP:

Theorem 3.6. $\operatorname{DRP}\left(\theta, I C_{\omega_{1}}\right)$ holds if and only if there are stationarily many $M \in$ $\wp \omega_{2}\left(H_{\left(\theta^{\omega}\right)^{+}}\right)$such that $M \cap H_{\theta} \in \mathrm{IC}_{\omega_{1}}$ and $N S \uparrow\left[H_{\theta}\right]^{\omega}$ condenses correctly via $M$.

Proof. Assume $\operatorname{DRP}\left(\theta, \mathrm{IC}_{\omega_{1}}\right)$. Consider any $M \in \wp_{\omega_{2}}\left(H_{\left(\theta^{\omega}\right)^{+}}\right)$from the stationary set which witnesses $\operatorname{DRP}\left(\theta, \mathrm{IC}_{\omega_{1}}\right)$. Let $\sigma_{M}: \bar{M} \rightarrow M \prec H_{\left(\theta^{\omega}\right)}+$ be the inverse of the Mostowski collapse of $M$, and $\bar{H}:=\sigma_{M}^{-1}\left(H_{\theta}\right)$. Let $\bar{R} \in \bar{M}$ and suppose $\bar{M} \models$ " $\bar{R}$ is a stationary subset of $[\bar{H}]^{\omega}$." We need to see that $\bar{R}$ really is stationary in $[\bar{H}]^{\omega}$ from the point of view of $V$. So let $\overline{\mathcal{A}}=\left(\bar{H},\left(\bar{f}_{n}\right)\right)_{n \in \omega} \in V$ be any algebra on $\bar{H}$. We need to find some $\bar{N} \in \bar{R}$ such that $\bar{N} \prec \overline{\mathcal{A}}$.

Let $R:=\sigma_{M}(\bar{R}) \in M$, and let $\mathcal{A}$ be the result of transferring the structure $\overline{\mathcal{A}}$ pointwise to $\sigma_{M} " \bar{H}=M \cap H_{\theta}$; i.e. $\mathcal{A}:=\left(M \cap H_{\theta},\left(f_{n}\right)\right)_{n \in \omega}$, where $f_{n}(y):=$ $\sigma_{M}\left(\bar{f}_{n}\left(\sigma_{M}^{-1}(y)\right)\right)$ for every $y \in M \cap H_{\theta}$. Note $R$ is stationary in $V$, since $M \prec$ $H_{\left(\theta^{\omega}\right)}+$. So $R \cap\left[M \cap H_{\theta}\right]^{\omega}$ is stationary, and by (2.2) there is some $N \in M \cap[M \cap$ $\left.H_{\theta}\right]^{\omega} \cap R$ such that $N \prec \mathcal{A}$. Note that $N$ is a countable element of $M$ and so $N \subset M=\operatorname{range}\left(\sigma_{M}\right)$; this ensures that $\bar{N}:=\sigma_{M}^{-1}(N)$ is closed under the functions in $\overline{\mathcal{A}}$. So $\bar{N} \in \bar{R}$ and $\bar{N} \prec \overline{\mathcal{A}}$.

For the converse, suppose $\operatorname{DRP}\left(\theta, \mathrm{IC}_{\omega_{1}}\right)$ fails; so for all but nonstationarily many $M \in \wp \omega_{2}\left(H_{\left(\theta^{\omega}\right)^{+}}\right)$such that $M \cap H_{\theta} \in \mathrm{IC}_{\omega_{1}}$ there is some $R \in M$ which is stationary in $\left[H_{\theta}\right]^{\omega}$ but $R \cap\left[M \cap H_{\theta}\right]^{\omega}$ is nonstationary. Let $\sigma_{M}: \bar{H} \rightarrow M$ be the inverse 
of the Mostowski collapse of $M, \bar{H}:=\sigma_{M}^{-1}\left(H_{\theta}\right)$, and $\bar{R}:=\sigma_{M}^{-1}(R)$. We show that $V \models$ " $\bar{R}$ is not stationary in $[\bar{H}]^{\omega}$ ". Let $\mathcal{A}=\left(M \cap H_{\theta},\left(f_{n}\right)\right)_{n \in \omega}$ be an algebra on $M \cap H_{\theta}$ which witnesses that $R \cap\left[M \cap H_{\theta}\right]^{\omega}$ is nonstationary. Similarly to the other direction of the proof, $\mathcal{A}$ can be transferred to an algebra $\overline{\mathcal{A}}$ on $\bar{H}$. If there were some $\bar{z} \in \bar{R}$ such that $\bar{z} \prec \overline{\mathcal{A}}$, then $\sigma_{M}(\bar{z}) \in R \cap\left[M \cap H_{\theta}\right]^{\omega}$ would be an elementary substructure of $\mathcal{A}$, which is impossible by the choice of $\mathcal{A}$. So $\overline{\mathcal{A}}$ witnesses that $\bar{R}$ is not stationary in $[\bar{H}]^{\omega}$.

The original version of this paper only showed the forward direction of Theorem 3.6 (that DRP implies condensation). We thank the anonymous referee for pointing out that the converse also holds.

Weak DRP implies a weaker form of condensation:

Theorem 3.7. $\operatorname{wDRP}\left(\theta, \operatorname{Unif}_{\omega_{1}}\right)$ implies that for every regular $\theta \geq \omega_{2}$, there are stationarily many $M \in \wp_{\omega_{2}}\left(H_{\left(\theta^{\omega}\right)}\right)$ such that $N S \uparrow(\theta \cap \operatorname{cof}(\omega))$ condenses correctly via $M$.

Proof. The proof is similar to the proof of Theorem 3.6, except (2.1) is used instead of (2.2). Consider any $M \in \wp_{\omega_{2}}\left(H_{\left(\theta^{\omega}\right)^{+}}\right)$from the stationary set in the conclusion of Lemma 3.3. Let $\sigma_{M}: \bar{M} \rightarrow M \prec H_{\left(\theta^{\omega}\right)+}$ be the inverse of the Mostowski collapse of $M$, and $\bar{\theta}:=\sigma_{M}^{-1}(\theta)$. Let $\bar{R} \in \bar{M}$ and suppose $\bar{M} \models " \bar{R}$ is a stationary subset of $\bar{\theta}$ ". We need to see that $\bar{R}$ really is stationary from the point of view of $V$. So let $\bar{C} \in V$ be any $\omega$-club in $\bar{\theta}$. By (2.1), $C:=\sigma_{M}$ " $\bar{C}$ is an $\omega$-club in $\sup (M \cap \theta)$. Let $R:=\sigma_{M}(\bar{R})$; note that $R$ is stationary in $\theta$ since $M \prec H_{\left(\theta^{\omega}\right)^{+}} . R$ reflects to $\sup (M \cap \theta)$, so $R \cap C \neq \emptyset$ and thus $\bar{R} \cap \bar{C} \neq \emptyset$.

DRP can be viewed as essentially reversing the first two quantifiers in the following reflection principle, now called the Weak Reflection Principle at $\theta(\operatorname{WRP}(\theta))$ :

For every stationary $R \subset[\theta]^{\omega}$, there exists a stationary $S_{R} \subset$ $\wp_{\omega_{2}}(\theta)$ such that for every $M \in S_{R}, R$ reflects at $[M]^{\omega}$.

$\operatorname{DRP}(\theta)$ implies $\operatorname{WRP}(\theta)$, and $\operatorname{WRP}(\theta)$ for $\theta \geq \omega_{2}$ follows from MM by $[7$.

If $Z$ is some class of structures which are not in $\operatorname{Unif}_{\omega_{1}}$, the author does not know if $\operatorname{DRP}(\theta, Z)$ implies $\operatorname{DRP}\left(\theta, \operatorname{Unif}_{\omega_{1}}\right)$; the answer is probably no, by arguments of Krueger [9].

Larson in [10] defined a principle he called $\mathrm{OSR}_{\omega_{2}}$, which is a version of DRP for stationary subsets of $\omega_{2} \cap \operatorname{cof}(\omega)$; he also showed that $\operatorname{OSR}_{\omega_{2}}$ follows from MM, but does not follow from SRP 2

Unlike SRP, DRP is consistent with $\mathrm{CH}$, because DRP holds in the model $V^{C o l\left(\omega_{1},<\kappa\right)}$ where $\kappa$ is a supercompact cardinal. The proof is the same as in 7,3

We show that a result of Foreman [6] concerning MM factors through wDRP. We first recall his theorem:

Theorem 3.8 (Foreman). Assume Martin's Maximum. Suppose that $\theta \geq \omega_{2}$ is regular and $H \subset H_{\theta}$ has cardinality $\theta$ and $\theta \subset H$. Let $\left\langle R_{x} \mid x \in H\right\rangle$ be a partition of $\theta \cap \operatorname{cof}(\omega)$ into stationary sets. Then there is a stationary subset $A \subset[H]^{<\omega_{2}}$ such that for all $N \in A: x \in N$ iff $R_{x}$ is stationary in $\sup (N \cap \theta)$.

\footnotetext{
${ }^{2} \operatorname{OSR}_{\omega_{2}}$ states: For every $\omega_{2}$ sequence $\left\langle R_{\alpha} \mid \alpha<\omega_{2}\right\rangle$ of stationary subsets of $\omega_{2} \cap \operatorname{cof}(\omega)$, there is a $\gamma \in \omega_{2} \cap \operatorname{cof}\left(\omega_{1}\right)$ such that for every $\alpha<\gamma, R_{\alpha}$ reflects at $\gamma$.

${ }^{3}$ Namely, if $\hat{j}: V^{\operatorname{Col}\left(\omega_{1},<\kappa\right)} \rightarrow M^{\operatorname{Col}\left(\omega_{1},<j(\kappa)\right)}$ is a lifting of a $\left(\theta^{\omega}\right)^{+}$-supercompact embedding

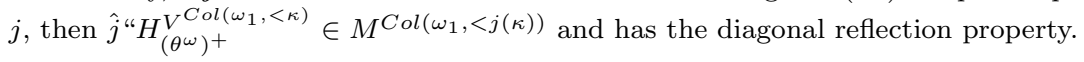


Theorem 3.9. ${ } \operatorname{DRP}\left(\theta, \operatorname{Unif}_{\omega_{1}}\right)$ implies the conclusion of Theorem 3.8 .

Proof. Let $H$ and $\vec{R}$ be a partition of $\theta \cap \operatorname{cof}(\omega)$ as in Theorem 3.8. Let $S$ be the stationary set from the conclusion of Lemma 3.3. Let $M \in S$ be such that $M \prec\left(H_{\left(\theta^{\omega}\right)^{+}}, \in, H, \vec{R}\right)$. Then $R_{x} \in M$ for every $x \in H \cap M$, so by Lemma 3.3. $R_{x}$ is stationary in $\sup (M \cap \theta)$.

If $x \in H-M$, we show that $R_{x}$ cannot reflect at $\sup (M \cap \theta)$. Now $M \cap H_{\theta} \in$ Unif $_{\omega_{1}}$, so $M \cap \theta$ is an $\omega$-closed unbounded set. But $R_{x}$ does not intersect $M \cap \theta$. Suppose there is some $\gamma \in R_{x} \cap M \cap \theta$; then since $\vec{R}$ is a partition, $x$ is definable from $\gamma$ in the structure $\left(H_{\left(\theta^{\omega}\right)^{+}}, \in, H, \vec{R}\right)$. Thus $x$ would be an element of $M$, a contradiction.

\section{DRP FOLLOWS FROM $P F A^{+\omega_{1}}$}

In this section we prove:

Theorem 4.1. $P F A^{+\omega_{1}}$ implies $\operatorname{DRP}\left(\theta, \mathrm{IA}_{\omega_{1}}\right)$ for every regular $\theta \geq \omega_{2}$.

In fact, just $M A^{+\omega_{1}}$ ( $\sigma$-closed) suffices. Let $\mathbb{P}$ be the $\sigma$-closed forcing for shooting an internally approachable sequence of length $\omega_{1}$ through $H_{\left(\theta^{\omega}\right)^{+}}$: conditions are internally approachable chains $\left\langle N_{\beta} \mid \beta \leq \delta\right\rangle$ of countable submodels of $H_{\left(\theta^{\omega}\right)^{+}}$, where $\delta<\omega_{1}$, and order is by end-extension 4 It is standard that if $G$ is generic for this poset and $H:=H_{\left(\theta^{\omega}\right)^{+}}^{V}$, then

$$
\bigcup G \text { witnesses that } H \in \mathrm{IA}_{\omega_{1}} \text {. }
$$

Set $N_{\alpha}^{G}:=G(\alpha)$. Note that $\left|H_{\left(\theta^{\omega}\right)^{+}}^{V}\right|^{V[G]}=\omega_{1}$; so there is a name $\left\langle\dot{R}_{\beta} \mid \beta<\omega_{1}\right\rangle$ such that $1_{\mathbb{P}} \Vdash$ " $\overrightarrow{\dot{R}}$ enumerates all stationary subsets of $[\theta]^{\omega}$ which are elements of $H$ (i.e. of the ground model)".

For each $\beta<\omega_{1}$ let $\dot{S}_{\beta}$ be the $\mathbb{P}$-name for $\left\{\alpha<\omega_{1} \mid \dot{N}_{\alpha}^{\dot{G}} \cap \theta \in \dot{R}_{\beta}\right\}$. Since $\mathbb{P}$ is $\sigma$ closed and thus proper, then $1_{\mathbb{P}} \Vdash$ " $\dot{R}_{\beta}$ is stationary in $[\theta] \omega$ ". Since $\left\{N_{\alpha}^{G} \mid \alpha<\omega_{1}\right\}$ is a club subset of $[H]^{\omega}$ for any generic $G$, then $1_{\mathbb{P}} \Vdash$ " $\dot{S}_{\beta}$ is stationary in $\omega_{1}$ ".

By Theorem 2.3 , there are stationarily many $M \in \wp_{\omega_{2}}\left(H_{\Omega}\right)$ (where $\Omega$ is sufficiently large) such that there is some $g_{M} \subset M$ which is $(M, \mathbb{P})$-generic and such that for all $\beta<\omega_{1},\left(\dot{S}_{\beta}\right)_{g_{M}}$ is stationary. Pick such an $M$ and a $\beta<\omega_{1}$. For each $\xi<\omega_{1}$ let $N_{\xi}^{g_{M}}$ abbreviate $\left(\dot{N}_{\xi}^{\dot{G}}\right)_{g_{M}}$; see Definition 2.4 for the meaning of the notation $\left(\dot{N}_{\xi}^{\dot{G}}\right)_{g_{M}}$. Note that (4.1) and Lemma 2.6 imply that $M \cap H \in \mathrm{IA}_{\omega_{1}}$, and that this is witnessed by $\left\langle N_{\xi}^{g_{M}} \mid \xi<\omega_{1}\right\rangle$. Since $\left(\dot{S}_{\beta}\right)_{g_{M}}$ is stationary, then $\left\{N_{\xi}^{g_{M}} \mid \xi \in\left(\dot{S}_{\beta}\right)_{g_{M}}\right\}$ is stationary in $\left[\bigcup_{\xi<\omega_{1}} N_{\xi}^{g_{M}}\right]^{\omega}=[M \cap H]^{\omega}$. Whenever $\xi \in\left(\dot{S}_{\beta}\right)_{g_{M}}$ there is some condition in $g_{M}$ which forces the $\Sigma_{0}$ statement $\dot{N}_{\xi}^{\dot{G}} \cap \theta \in \dot{R}_{\beta}$ (and that these objects are in $H$ ); so by Lemma 2.5. $N_{\xi}^{g_{M}} \cap \theta \in\left(\dot{R}_{\beta}\right)_{g_{M}}$. This shows that $\left(\dot{R}_{\beta}\right)_{g_{M}}$ reflects to $M \cap \theta$.

The $(M, \mathbb{P})$-genericity of $g_{M}$ implies that $\left\langle\left(\dot{R}_{\beta}\right)_{g_{M}} \mid \beta<\omega_{1}\right\rangle$ enumerates all stationary subsets of $[\theta]^{\omega}$ which are elements of $M$. Thus $M$ has the desired property.

\footnotetext{
${ }^{4}$ If $\left\langle N_{\beta} \mid \beta \leq \delta\right\rangle$ is a $\in$-increasing and $\subset$-continuous chain, where $\delta$ is countable, we say the chain is internally approachable iff $\vec{N}\left\lceil\delta \in N_{\delta}\right.$. Note that this can only happen if $\delta$ is a successor ordinal.
} 


\section{MARTIN'S MAXIMUM IMPLIES WDRP}

In this section we prove:

Theorem 5.1. MM implies $\operatorname{wDRP}\left(\theta, \mathrm{IA}_{\omega_{1}}\right)$ for every regular $\theta \geq \omega_{2}$.

The forcing and proof here are based on the proof of Theorem 34 of [6]. Assume MM. Fix a partition $\left\langle T_{\alpha} \mid \alpha<\omega_{1}\right\rangle$ of $\omega_{1}$ such that:

- $T_{\alpha} \cap T_{\beta}=\emptyset$ for every $\alpha \neq \beta$.

- $\nabla \alpha<\omega_{1} T_{\alpha}$ contains all limit ordinals in $\omega_{1}$ (so, in particular, $\vec{T}$ is a maximal antichain).

Such a partition exists as a consequence of ZFC.

Define a partial order $\mathbb{Q}=\mathbb{Q}_{\vec{T}}$; conditions are pairs $\left(f,\left\langle N_{\alpha} \mid \alpha \leq \delta\right\rangle\right)$ such that:

(1) $\delta<\omega_{1}$;

(2) $\vec{N}$ is an $\in$-increasing and $\subset$-continuous chain of countable elementary substructures of $H_{\left(\theta^{\omega}\right)}$;

(3) $f: \delta+1 \rightarrow H_{\left(\theta^{\omega}\right)+}$;

(4) for every $\beta<\delta$ : if $f(\beta)$ is a projective stationary subset of $[\theta]^{\omega}$, then for all limits $\beta^{\prime} \in T_{\beta} \cap(\beta, \delta]: N_{\beta^{\prime}} \cap \theta \in f(\beta)$.

The order $\leq_{\mathbb{Q}}$ is by end-extension. If $q$ is a condition, then $f^{q}$ and $\vec{N}^{q}$ denote the first and second coordinates, respectively, of $q$, and $\delta^{q}$ denotes the ordinal such that $\delta^{q}+1=\operatorname{domain}\left(f^{q}\right)=\operatorname{domain}\left(\vec{N}^{q}\right)$. In all the following arguments, if $N \prec H_{\left(\theta^{\omega}\right)^{+}}$ is countable, then $\delta_{N}:=N \cap \omega_{1}$.

Lemma 5.2. For $a \in H_{\left(\theta^{\omega}\right)^{+}}$, let $D_{a}$ be the set of conditions $q$ such that $a \in$ range $\left(f^{q}\right) \cap N_{\delta^{q}}^{q}$ and $\vec{N}^{q} \uparrow \delta^{q} \in N_{\delta^{q}}^{q}$. Then $D_{a}$ is dense.

Proof. This is because there are essentially no requirements for the models indexed by successor ordinals in the conditions. Let $a \in H_{\left(\theta^{\omega}\right)}$. Let $q=\left(f^{q}, \vec{N}^{q}\right)$ (recall the domain of $f^{q}$ and $\vec{N}^{q}$ is $\left.\delta^{q}+1\right)$. Pick any $N^{\prime}$ such that $N^{\prime} \prec\left(H_{\left(\theta^{\omega}\right)^{+}}, \in,\{a, q\}\right)$, and let $q^{\prime}:=q^{\wedge}\left\langle\left(a, N^{\prime}\right)\right\rangle$. Then $q^{\prime}$ is a condition, since $N^{\prime}$ is indexed by the successor ordinal $\delta^{q}+1$ and therefore has no requirements regarding any of the previous $f^{q^{\prime}}(\beta)^{\prime}$ 's. Also, $\overrightarrow{N^{q^{\prime}}}\left\lceil\delta^{q^{\prime}}=\vec{N}^{q^{\prime}}\left\lceil\delta^{q}+1=\vec{N}^{q} \in N^{\prime}=N_{\delta^{q^{\prime}}}^{q^{\prime}}\right.\right.$ and $a \in N^{\prime}=N_{\delta^{q^{\prime}}}^{q^{\prime}}$.

Lemma 5.3. For every $\beta<\omega_{1}$, the set $\{q \in \mathbb{Q} \mid \beta \in \operatorname{domain}(q)\}$ is dense.

Proof. We prove the statement of the lemma by induction on $\beta$. Suppose $\beta$ is a successor ordinal, say $\beta=\bar{\beta}+1$. Let $q \in \mathbb{Q}$. By the induction hypothesis, without loss of generality, $\bar{\beta} \in \operatorname{domain}(q)$. Similarly to the proof of Lemma $5.2, q$ can be extended to a condition $q^{\prime}$ which has $\beta=\bar{\beta}+1$ in its domain, since there are virtually no requirements on what $f(\bar{\beta}+1), N_{\bar{\beta}+1}$ should be.

Now suppose $\beta$ is a limit ordinal. Let $q \in \mathbb{Q}$ be arbitrary. Since $\nabla \alpha<\omega_{1} T_{\alpha}$ contains all limit ordinals, there is a (unique) $\alpha^{*}<\beta$ such that $\beta \in T_{\alpha^{*}}$. Fix an increasing sequence $\left\langle\beta_{n} \mid n \in \omega\right\rangle$ cofinal in $\beta$, such that $\alpha^{*}<\beta_{0}$. By the induction hypothesis, without loss of generality, $\alpha^{*} \in \operatorname{domain}(q)$.

There are stationarily many countable $N \prec H_{\left(\theta^{\omega}\right)}$ such that $q, \vec{\beta} \in N$ and, if $f^{q}\left(\alpha^{*}\right)$ is a stationary set, $N \cap \theta \in f^{q}\left(\alpha^{*}\right)$ Fix any such $N$, and let $\left\{y_{n} \mid n \in \omega\right\}$ be some enumeration of $N$. Recursively define a descending sequence of conditions as follows: set $q_{0}:=q(\in N)$. Given $q_{n-1}$, applying the induction hypothesis and

${ }^{5}$ Note that this uses only stationarity, not projective stationarity, of $f^{q}\left(\alpha^{*}\right)$. 
Lemma 5.2 inside $N$ yields some condition $q_{n} \in N$ such that $\beta_{n} \in \operatorname{dom}\left(q_{n}\right) \sqrt[6]{6} q_{n} \leq$ $q_{n-1}$, and $y_{n} \in N_{\delta^{q_{n}}}^{q_{n}}$. Then $\sup _{n \in \omega} \operatorname{dom}\left(q_{n}\right) \geq \beta$. If $\sup _{n \in \omega} \operatorname{dom}\left(q_{n}\right)>\beta$, then $\beta$ is an element of $\operatorname{dom}\left(q_{n}\right)$ for some $n$ and this finishes the proof. So we can assume now that $\sup _{n \in \omega} \operatorname{dom}\left(q_{n}\right)=\beta$. Set $f:=\left(\bigcup_{n \in \omega} f^{q_{n}}\right)^{\wedge}\langle\emptyset\rangle$ and $\vec{N}:=\left(\bigcup_{n \in \omega} \vec{N}^{q_{n}}\right)^{\wedge}\langle N\rangle$; we show that $(f, \vec{N})$ is a condition. Note that the domain of both $f$ and $\vec{N}$ is $\beta+1$; so $N$ is indexed by $\beta$ in the sequence $\vec{N}$. Recall that we chose $N$ so that $N \cap \theta \in f^{q}\left(\alpha^{*}\right)$ (if $f^{q}\left(\alpha^{*}\right)$ is stationary in $\left.[\theta]^{\omega}\right)$; and by the pairwise disjointness of $\vec{T}, f^{q}\left(\alpha^{*}\right)$ is the only stationary set in the range of $f$ which $N \cap \theta$ must belong to. Finally, since $y_{n} \in N_{\delta q_{n}}^{q_{n}}$ for each $n$, then $N$ is the $\subset$-limit of the models in the $q_{n}$.

Lemma 5.4. $\mathbb{Q}$ is stationary set-preserving.

Proof. Let $S \subset \omega_{1}$ be stationary and $q \Vdash$ " $\dot{C}$ is a club subset of $\omega_{1}$ ". Since $\vec{T}$ is a maximal antichain there is a $\beta_{S}<\omega_{1}$ such that $S \cap T_{\beta_{S}}$ is stationary.

By Lemma 5.3, without loss of generality, $\beta_{S} \in \operatorname{domain}(q)$. Let $R^{*}:=f^{q}\left(\beta_{S}\right)$. Pick an $N$ such that $\delta_{N} \in S \cap T_{\beta_{S}}, N \prec\left(H_{\left(\theta^{\omega}\right)^{+}}, \in,\{\dot{C}, q\}\right)$, and if $R^{*}$ is a projective stationary subset of $[\theta]^{\omega}$ also require $N \cap \theta \in R^{*}$. Let $\left\{y_{n} \mid n \in \omega\right\}$ be an enumeration of $N$, and fix a cofinal sequence $\left\langle\delta_{n} \mid n \in \omega\right\rangle$ cofinal in $\delta_{N}$. Inductively build a sequence of conditions $q=q_{0} \geq q_{1} \geq q_{2} \geq \ldots$ such that for all $n \in \omega$ :

(i) $q_{n} \in N$;

(ii) $\delta_{n} \in$ domain $\left(q_{n}\right)$ and $y_{n} \in \operatorname{range}\left(f^{q_{n}}\right)$ (this uses Lemmas 5.2 and 5.3);

(iii) $q_{n} \Vdash \dot{C} \cap\left[\delta_{n}, \delta_{N}\right) \neq \emptyset$.

Note that $\left.\delta_{N}=\operatorname{dom}\left(\bigcup_{n \in \omega} f^{q_{n}}\right)=\operatorname{dom}\left(\bigcup_{n \in \omega} \vec{N}^{q_{n}}\right)\right]$ Define $f^{*}$ as $\left(\bigcup_{n \in \omega} f^{q_{n}}\right)^{\wedge}\langle\emptyset\rangle$, $\vec{N}^{*}$ as $\left(\bigcup_{n \in \omega} \vec{N}^{q_{n}}\right)^{\wedge}\langle N\rangle$, and $q^{*}$ as $\left(f^{*}, \vec{N}^{*}\right)$. Then $q^{*}$ is a condition: $\beta_{S}$ is the unique $\beta$ such that $\delta_{N} \in T_{\beta}$, and $N \cap \theta$ is in $f^{q^{*}}\left(\beta_{S}\right)=f^{q}\left(\beta_{S}\right)$ (if this is a projective stationary set) by our choice of $N$. Also, (ii) implies that $N$ is the $\subset$-limit of the models in the $q_{n}$ 's. Finally, (iii) implies that $q^{*} \Vdash " \check{\delta}_{N}$ is a limit of the club $\dot{C}$," so $q^{*} \Vdash \check{S} \cap \dot{C} \neq \emptyset$.

Therefore, by Theorem 2.3. there are stationarily many $M \in \wp_{\omega_{2}}\left(H_{\Omega}\right)$ such that $\omega_{1} \subset M$ and an $(M, \mathbb{Q})$-generic exists. Fix such an $M$ and a generic $g_{M} \subset M$; say $f^{g_{M}}$ is the evaluation of the first coordinate of $g_{M}$ and $\vec{N}^{g_{M}}$ is the evaluation of the second coordinate of $g_{M}$. Lemma 5.2 implies that there are densely many conditions which are internally approachable. So $1_{\mathbb{Q}} \Vdash " \check{H} \in \mathrm{IA}_{\omega_{1}}$ ", where $H:=H_{\left(\theta^{\omega}\right)^{+}}$; then Lemma2.6implies $M \cap H \in \mathrm{IA}_{\omega_{1}}$ and that this is witnessed by $\vec{N}^{g_{M}}$. Let $R \in M$ be a projective stationary subset of $[\theta]^{\omega}$. By Lemma 5.2 and genericity of $g_{M}$, there is a $\beta_{R}<\omega_{1}$ such that $f^{g_{M}}\left(\beta_{R}\right)=R$. Then for every limit $\beta^{\prime} \in T_{R}:=\left(\beta_{R}, \omega_{1}\right) \cap T_{\beta_{R}}$, $N_{\beta^{\prime}}^{g_{M}} \cap \theta \in R$; thus $R \cap[M \cap \theta]^{\omega}$ contains the stationary set $\left\{N_{\beta^{\prime}}^{g_{M}} \cap \theta \mid \beta^{\prime} \in T_{R}\right\}$. This completes the proof of Theorem 5.1 .

\section{Some Questions}

We end with some questions.

(1) Let $Z$ be a class of $\omega_{1}$-sized structures (e.g. $\left.Z=\operatorname{IA}_{\omega_{1}}\right)$, and let $\operatorname{RP}_{\omega_{1}}(\theta, Z)$ denote the statement: "For every $\omega_{1}$-sized collection $\mathcal{S}$ of stationary subsets

\footnotetext{
${ }^{6}$ Recall that we required $\vec{\beta} \in N$, so in particular each $\beta_{n} \in N$.

${ }^{7}$ This is because $\delta_{n} \in \operatorname{dom}\left(f^{q_{n}}\right)$ by (ii), and $\operatorname{dom}\left(f^{q_{n}}\right)<\delta_{N}$ by (i).
} 
of $[\theta]^{\omega}$, there are stationarily many $M \in \wp_{\omega_{2}}\left(H_{\left(\theta^{\omega}\right)^{+}}\right)$such that $M \cap H_{\theta} \in Z$ and every $S \in \mathcal{S}$ reflects at $[M \cap \theta]^{\omega}$ " (i.e. simultaneous reflection of $\omega_{1}$ many stationary sets).

Clearly $\operatorname{DRP}(\theta)$ implies $\operatorname{RP}_{\omega_{1}}(\theta)$. Does the converse hold?

(2) Does MM imply $\operatorname{DRP}(\theta, Z)$ for any $\theta \geq \omega_{2}$ (where $Z$ is, say, $\left.\operatorname{Unif}_{\omega_{1}}\right)$ ?

(3) Is there a consistent, natural diagonal version of the Strong Reflection Principle?

(4) Does the consequence of Theorem 3.8 imply wDRP?

\section{REFERENCES}

[1] James E. Baumgartner, A new class of order types, Ann. Math. Logic 9 (1976), no. 3, 187222. MR0416925 (54:4988)

[2] _ Applications of the proper forcing axiom, Handbook of Set-Theoretic Topology, North-Holland, Amsterdam, 1984, pp. 913-959. MR776640 (86g:03084)

[3] Sean Cox, Covering theorems for the core model, and an application to stationary set reflection, Ann. Pure Appl. Logic 161 (2009), no. 1, 66-93. MR2567927

[4] Qi Feng and Thomas Jech, Projective stationary sets and a strong reflection principle, J. London Math. Soc. (2) 58 (1998), no. 2, 271-283. MR.1668171(2000b:03166)

[5] Matthew Foreman, Ideals and Generic Elementary Embeddings, Handbook of Set Theory, Springer, 2010

[6] - Smoke and mirrors: combinatorial properties of small cardinals equiconsistent with huge cardinals, Adv. Math. 222 (2009), no. 2, 565-595. MR.2538021

[7] M. Foreman, M. Magidor, and S. Shelah, Martin's maximum, saturated ideals, and nonregular ultrafilters. I, Ann. of Math. (2) 127 (1988), no. 1, 1-47. MR924672 (89f:03043)

[8] Leo Harrington and Saharon Shelah, Some exact equiconsistency results in set theory, Notre Dame J. Formal Logic 26 (1985), no. 2, 178-188. MR783595 (86g:03079)

[9] John Krueger, On the weak reflection principle, to appear in Transactions of the American Mathematical Society.

[10] Paul Larson, Separating stationary reflection principles, J. Symbolic Logic 65 (2000), no. 1, 247-258. MR1782117 (2001k:03094)

[11] Menachem Magidor, Reflecting stationary sets, J. Symbolic Logic 47 (1982), no. 4, 755-771 (1983). MR683153 (84f:03046)

[12] W. Hugh Woodin, The axiom of determinacy, forcing axioms, and the nonstationary ideal, de Gruyter Series in Logic and its Applications, vol. 1, Walter de Gruyter \& Co., Berlin, 1999. MR1713438(2001e:03001)

Institut Für Mathematische Logik und GrundLAGenforschung, Universität MÜNSter, Einsteinstrasse 62, 48149 Münster, Germany

E-mail address: sean.cox@uni-muenster.de 